\title{
Alterstice
}

Revue internationale de la recherche interculturelle

International Journal of Intercultural Research

Revista International de la Investigacion Intercultural

\section{Le développement de programmes de prévention pour les enfants immigrants et réfugiés : douter de l'évidence}

\section{Cécile Rousseau}

\section{Volume 3, numéro 1, 2013}

Les diversités au coeur de la recherche interculturelle : vers de nouvelles perspectives

URI : https://id.erudit.org/iderudit/1077499ar

DOI : https://doi.org/10.7202/1077499ar

Aller au sommaire du numéro

Éditeur(s)

Alterstice

ISSN

1923-919X (numérique)

Découvrir la revue

Citer cet article

Rousseau, C. (2013). Le développement de programmes de prévention pour les enfants immigrants et réfugiés : douter de l'évidence. Alterstice, 3(1), 61-66. https://doi.org/10.7202/1077499ar
Résumé de l'article

À partir d'une réflexion sur les prémisses pouvant guider le développement de programmes de prévention pour les enfants immigrants et réfugiés, ce texte souhaite relancer le débat autour de l'utilité et des limites de l'approche proposée par les données probantes. Le travail avec les enfants immigrants et réfugiés, en tant que porteurs de différence, remet en question les savoirs experts et met en évidence la violence possible des processus de normalisation et de standardisation qu'ils ont tendance à promouvoir. Le défi est alors de réintroduire une complexité qui tienne compte de la singularité des sujets et des forces collectives dans des processus de développement de programmes de prévention. Ceux-ci doivent être ancrés dans une réalité spécifique, sans toutefois faire l'économie de la rigueur. 


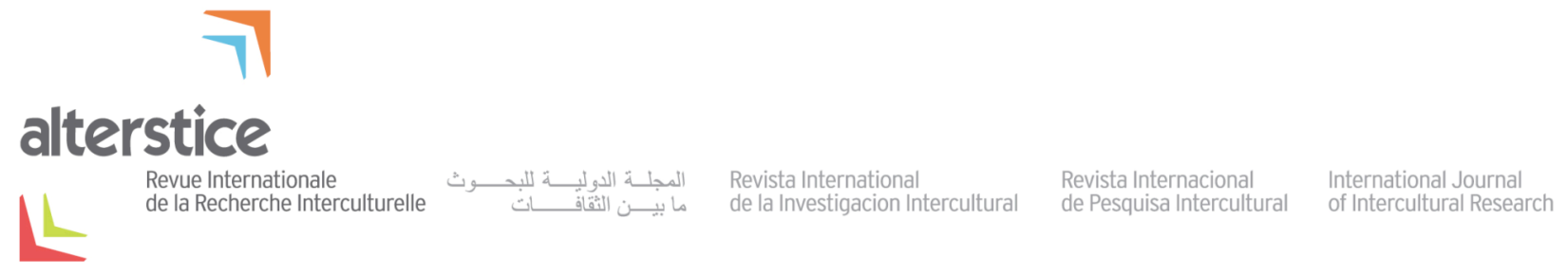

ARTICLE THÉMATIQUE

\section{Le développement de programmes de prévention pour les enfants immigrants et réfugiés : douter de l'évidence}

Cécile Rousseau ${ }^{1}$

\section{Résumé}

À partir d'une réflexion sur les prémisses pouvant guider le développement de programmes de prévention pour les enfants immigrants et réfugiés, ce texte souhaite relancer le débat autour de l'utilité et des limites de l'approche proposée par les données probantes. Le travail avec les enfants immigrants et réfugiés, en tant que porteurs de différence, remet en question les savoirs experts et met en évidence la violence possible des processus de normalisation et de standardisation qu'ils ont tendance à promouvoir. Le défi est alors de réintroduire une complexité qui tienne compte de la singularité des sujets et des forces collectives dans des processus de développement de programmes de prévention. Ceux-ci doivent être ancrés dans une réalité spécifique, sans toutefois faire l'économie de la rigueur.

\section{Rattachement de l'auteure \\ ${ }^{1}$ Université McGill, Montréal, Canada}

\section{Correspondance}

cecile.rousseau@mcgill.ca

\section{Mots clés}

prévention; enfants immigrants et réfugiés; données probantes; santé mentale

\section{Pour citer cet article :}

Rousseau, C. (2013). Le développement de programmes de prévention pour les enfants immigrants et réfugiés: douter de l'évidence. Alterstice, 3(1), 61-66. 


\section{Introduction}

Guidant les prises de décisions administratives et cliniques et modulant l'obtention de fonds et l'orientation des grands programmes de prévention, le credo de la rigueur scientifique s'est incarné dans l'idéologie des données probantes. Cette idéologie propose un parcours méthodologique de construction des connaissances qui se situe en droite ligne avec l'héritage de la philosophie des sciences, de Descartes à Popper (Bourgeault, 1999). II s'agit là d'une démarche extrêmement utile pour remettre en question des pratiques fondées sur la tradition et les habitudes et pour promouvoir et développer de nouveaux programmes, entre autres dans le domaine de la prévention. Par contre, au-delà de l'exigence méthodologique, l'interprétation des résultats des études cliniques randomisées pose de nombreux problèmes. Par exemple, la distinction entre la signification statistique et l'efficacité clinique dans des environnements contrôlés n'est souvent pas faite (NICE, 2005), pas plus que la distinction entre l'efficacité en contexte de recherche et l'efficience dans des milieux de vie divers. Le désir de certitude, imposé par la prescription sociale de compétence, pousse à la réification des fragments de savoir produits, faisant largement fi de la complexité singulière et sociale de l'humain. Les modèles standardisés qui en résultent peuvent donner des résultats intéressants, mais ils sont trop souvent une source de contrainte pour les intervenants qui essayent de les adapter de façon discrète à leur réalité quotidienne, quand ils ne deviennent pas un fardeau pour les populations qu'ils prétendent aider.

Afin d'illustrer ce processus et de réfléchir à des pistes permettant de réintroduire la complexité dans le développement de programmes de prévention, sans pour autant faire l'économie de la rigueur, je propose dans ce texte une brève réflexion sur les programmes de prévention s'adressant aux enfants immigrants et réfugiés. En effet, ces enfants et leurs familles incarnent l'«autre " porteur de différences déstabilisantes exigeant une décentration personnelle et institutionnelle. À ce titre, ils jouent un rôle important de révélateur des limites des processus de standardisation, soulignant, parfois de façon caricaturale, la nécessité incontournable de tenir compte de l'environnement social et culturel dans le développement de programmes de prévention. Sans faire une revue exhaustive des problèmes que peut poser l'application de programmes conçus et évalués auprès des enfants de la majorité à des communautés minoritaires, je m'attarderai sur trois aspects : la spécificité des processus de risque et de protection pour les enfants migrants et réfugiés, la question de l'adaptation culturelle des programmes basés sur les données probantes et la nécessité de concevoir des programmes alternatifs, fondés sur une vision écosystémique, qui pense la prévention en fonction d'une expérience de vie dans un environnement social, politique et culturel spécifique.

\section{Les processus de risque et de protection pour les enfants migrants et réfugiés}

Malgré une exposition à l'adversité souvent importante, les enfants immigrants et réfugiés ne présentent en moyenne pas plus de problèmes de santé mentale que leurs pairs de la société hôte, même s'ils se distinguent de ces derniers sur le plan de la présentation des symptômes, du sens qui leur est attribué ainsi que des trajectoires de recherche d'aide (Goodman, Patel et Leon, 2008). En Amérique du Nord, les enquêtes épidémiologiques récentes qui comparent les groupes ethniques en termes de risque de présenter un trouble psychiatrique de l'enfant soulignent surtout les similitudes au niveau de la prévalence des différents troubles (Roberts, Roberts et Xing, 2006). Les quelques différences entre groupes ethniques mises en évidence dans ces enquêtes disparaissent lorsque l'on contrôle pour le niveau de dysfonction et les facteurs associés. Par contre, la différence entre enfants immigrants et réfugiés et enfants appartenant à la majorité ressort lorsque l'on considère ce qui constitue pour eux des facteurs de risque ou de protection. Dans le cadre d'une méta analyse, Stevens et Volleger (2008) concluent qu'il n'est pas possible d'arriver à établir une relation directe entre la migration et le risque pour la santé mentale des enfants et qu'il faut tenir compte plutôt de la complexité des interactions entre les caractéristiques du groupe d'appartenance de l'enfant et la société hôte. Il ne s'agit pas de relations linéaires et des variations importantes entre la première génération et les générations subséquentes sont rapportées. Ainsi, le statut économique faible des familles appartenant à des minorités ne semble pas affecter directement l'état de santé mentale de leurs enfants (Beiser, Hou, Hyman et Tousignant, 2002). Au Québec, nos travaux ont documenté que les adolescents récemment immigrés, originaires des Caraïbes anglophones, présentent moins de problèmes émotionnels et de

comportements que leurs pairs qui sont au Québec depuis plus longtemps ou que ceux qui sont nés dans le pays hôte de familles originaires des Caraïbes (Rousseau, Hassan, Measham et Lashley, 2008). Le risque est alors associé

Alterstice - Revue Internationale de la Recherche Interculturelle, vol. 3, $n^{\circ} 1$ 
à l'interaction entre la protection que procure la cohésion familiale et la vulnérabilité qui provient à la fois de l'exposition à la discrimination et du sentiment d'impuissance face à des formes d'exclusion plus structurelle qui sont elles-mêmes en constante évolution. Ainsi, la détérioration du climat international, cristallisée autour d'évènements frappants comme le 11 septembre ou les guerres d'Afghanistan et d'Irak, a transformé la représentation de l' « autre » et exacerbé les tensions intercommunautaires, faisant de tout groupe minoritaire une menace potentielle (Hörnqvist, 2004). Ceci se traduit pour les populations immigrantes par un climat de plus en plus hostile (Rousseau, Hassan, Moreau et Thombs, 2010). Les pertes associées à la migration sont alors aggravées par l'image négative renvoyée par la majorité vers les minorités, ce qui a d'importantes implications pour l'identité et la santé mentale des enfants d'immigrants (Rousseau et Jamil, 2010). Chaque contexte d'accueil est caractérisé par des tensions spécifiques : la question raciale aux États-Unis et le débat sur la laïcité de l'école et le port du voile en Europe puis au Canada. Au Québec, c'est la question linguistique qui fait l'objet de débats passionnés (Bouchard et Taylor, 2008). Les résultats de l’Enquête sur la diversité ethnique démontrent qu'au Québec les minorités visibles dont la langue maternelle est l'anglais rapportent davantage de discrimination que celles dont la langue est le français et qu'elles associent la discrimination à la langue ou l'accent contrairement au reste du Canada ou la discrimination est plus souvent attribuée à des questions de phénotype ou de couleur de la peau (Bourhis, Montreuil, Helly et Jantzen, 2007). Ces déterminants locaux des relations majorité-minorités interagissent de façon complexe avec des éléments du contexte international et approfondissent le fossé des valeurs et des représentations sociales entre l'école et la maison, ce qui engendre une souffrance psychologique et des problèmes d'image de soi pour les enfants des minorités. En soulignant le rôle des facteurs sociaux et familiaux, ces travaux questionnent la médicalisation et la psychologisation des conséquences de l'immigration qui surgissent dans le cadre d'une tendance plus large à traduire en termes de pathologie la souffrance engendrée par des problèmes sociaux (Kleinman et Kleinman, 1997). En ce qui concerne les enfants, il importe de resituer les sources de souffrance sociale pré, péri et post-migratoire des jeunes issus de l'immigration dans une dynamique ou les enjeux du monde local de l'enfant sont en tension avec une transformation plus mondiale. Bien sûr, la question de l'appropriation du pouvoir par les parents au travers de pratiques n'est pas spécifique aux familles appartenant à des minorités, mais ces relations de pouvoir sont au cœur des interactions familles immigrantes - écoles et structurent largement la construction du lien social pour les enfants. Pour s'adresser à l'asymétrie de pouvoir entre les communautés immigrantes minoritaires et l'institution qu'est l'école, il faut transformer la perception que les écoles ont de ces communautés et miser sur l'hétérogénéité comme source de démocratie. C'est autour du racisme et de ses conséquences en termes d'isolement social et d'atteinte à l'identité (Tauvel, 1997) que le milieu scolaire a le plus proposé des pistes de solutions telles qu'une pédagogie antiraciste, une meilleure représentation des minorités dans le corps professoral, ou encore une éducation à la citoyenneté et à la justice sociale (Lin, 2001). Potvin, McAndrew et Kanoute (2006) ont évalué le degré de mise en œuvre de perspectives ou d'intervention relevant de l'éducation antiraciste dans les milieux scolaires francophones au Québec. Leurs résultats décrivent la présence de nombreuses initiatives valables dans le système scolaire, même si celles-ci sont encore loin de constituer un cadre de référence global. Les chercheurs soulignent que le rôle des pratiques éducatives dans le maintien, voir la production, de rapports inégalitaires est minimisé ou nié. Le rôle de l'école peut être conceptualisé comme un espace intermédiaire, point de rencontre entre la société et la maison (entre la culture d'origine et celle de la société hôte, entre diverses communautés d'appartenance). Cet espace met en jeu les liens entre le monde de l'enfant (celui qui lui est personnel et celui qui lui est transmis) et le monde externe (Winnicott, 1975). Les programmes de prévention et d'intervention devraient miser sur cette position de médiation de l'école qui lui confère un potentiel de transformation, non seulement sur le plan du développement de l'enfant, mais aussi pour contrer les dynamiques d'exclusion en devenant un lieu facilitant une certaine appropriation du pouvoir pour les familles et les jeunes, pour faciliter la rencontre de l'autre et le vivre ensemble et enfin pour soutenir l'intégration d'un passé souvent lourd de pertes et de traumatismes en ouvrant des espaces d'expression.

On comprendra dès lors qu'une transposition directe de programmes de prévention développés pour la majorité laisse dans l'ombre plusieurs des enjeux cruciaux pour les enfants immigrants, en inférant que ce qui constitue un risque ou une protection pour ces derniers est similaire à ce qui vulnérabilise ou protège leurs pairs de la société hôte. 


\section{Les dilemmes de l'adaptation culturelle}

Afin de s'atteler au problème de l'inadéquation pour des groupes minoritaires des programmes d'intervention ou de prévention développés largement auprès des majorités en Amérique du Nord et en Europe, plusieurs travaux ont proposé d'adapter culturellement des pratiques ayant une efficacité démontrée. II s'agit là d'une initiative intéressante dans la mesure où elle témoigne d'une prise de conscience du caractère culturellement spécifique des interventions proposées. Trop souvent cependant, l'adaptation suggérée est considérée comme étant de nature cosmétique et devient une carte de séduction au service de la dissémination des savoirs experts de la majorité. Ainsi, Ngo et collab. (2008) illustrent comment des interventions cognitivo-comportementales en milieu scolaire peuvent être adaptées culturellement pour des enfants appartenant à des minorités. D'après ces auteurs, l'adaptation culturelle est un processus d'« emballage " qui n'altère en rien les éléments actifs centraux de la thérapie cognitive-comportementale, ce qui démontre, à leur avis, qu'il est possible d'adapter culturellement ce type d'intervention dont l'efficacité a été démontrée par des données probantes. Cette conclusion soulève cependant des questions scientifiques et éthiques importantes.

Tout d'abord, afin d'« emballer » la thérapie, les auteurs disent qu'ils ont ajouté des pratiques artistiques (poèmes, écriture, arts visuels), religieuses (méditation, prières, rituels) et traditionnelles (brûler des herbes ayant une signification spéciale par exemple). Tous ces ajouts sont, en eux-mêmes, des formes d'intervention qui ont certes une dimension cognitive, mais qui ne peuvent être réduites à celle-ci, étant donné qu'ils mobilisent des processus communautaires, créatifs et spirituels que l'on retrouve souvent d'une façon ou d'une autre dans les grandes traditions de guérison tout au long de l'histoire de l'humanité. II existe bien sûr des convergences entre ces traditions et les psychothérapies contemporaines, ces dernières faisant par exemple des emprunts significatifs à certaines formes bouddhistes de méditation et d'éveil de la conscience (Hinton et collab., 2005). Toutefois, prétendre que, dans l'amalgame entre ces pratiques et la thérapie cognitivo-comportementale, seule cette dernière a des effets significatifs est erroné d'un point de vue méthodologique, étant donné que chacun des ajouts peut en lui-même avoir un effet thérapeutique. De plus, assigner l'efficacité au protocole établi par des savoirs experts associés à la culture des majorités et minimiser d'emblée la signification de ces pratiques alternatives constitue une forme d'ethnocentrisme, ou les savoirs autres sont récupérés sans que les formes autorisées de savoir aient besoin de se remettre en question.

\section{À la recherche d'alternatives}

Comment dès lors peut-on développer des programmes de prévention pour les enfants migrants et réfugiés? S'il n'existe pas de réponse simple, l'attention à des micropratiques prometteuses développées dans divers environnements peut fournir des pistes encourageant la créativité.

Les activités d'expression créatrice constituent un outil intéressant pour travailler avec les enfants immigrants sur la construction du sens autour de leur expérience et élaborer les enjeux liés à la multiplicité des identités ou à un vécu de discrimination raciale (Rousseau, Singh, Lacroix, Bagilishya et Measham, 2004). Dans le cas des enfants réfugiés, les activités créatrices facilitent l'élaboration des pertes multiples et des traumatismes, directs ou transmis de façon transgénérationnelle. Depuis 15 ans, notre équipe a développé et évalué des programmes qui misent sur la créativité pour soutenir les processus de transformation qui surgissent face à l'adversité et créer des ponts entre les multiples univers et les diverses identités des jeunes. Ces travaux se sont initialement fondés d'une part sur les recherches qui soulignaient la spécificité des profils de risque et de protection pour les jeunes migrants et réfugiés et, d'autre part, sur des recherches cliniques et qualitatives soulignant le rôle protecteur de la créativité pour ces jeunes. Le premier programme que nous avons développé s'adresse aux enfants du primaire et utilise des contes et des mythes comme métaphores structurantes pour relancer ou favoriser le travail de l'imaginaire chez des enfants récemment arrivés. II est associé à une diminution des symptômes et à une augmentation de l'estime de soi (Rousseau, Lacroix, Singh, Gauthier et Benoit, 2005). Des ateliers d'expression théâtrale s'adressant aux adolescents et une intervention utilisant le jeu de sable pour des enfants d'âge préscolaire ont par la suite été développés. Deux essais randomisés indiquent qu'ils sont efficaces au niveau de la réduction des symptômes ou du handicap associé (Rousseau et collab., 2007 et Rousseau, Benoit, Lacroix et Gauthier, 2009). Chacun de ces programmes a été développé par un processus d'essai-erreur sur plusieurs années. Toute proposition d'activités était évaluée à la fois par les jeunes eux-mêmes, au travers de ce qu'ils en disaient mais aussi de ce qu'ils 
exprimaient de multiples façons, par les enseignants et par les membres de l'équipe. Les programmes résultants sont des co-constructions, élaborées à plusieurs voix. Les évaluations quantitatives subséquentes ne viennent pas confirmer des savoirs experts, mais plutôt jeter un autre éclairage sur ce que les évaluations qualitatives ont révélé. Le succès de ces programmes de prévention confirme l'intérêt de développer des interventions alternatives, sensibles à la culture et au contexte sans pour autant renoncer à les soumettre à une évaluation rigoureuse. Le développement d'alternatives aux programmes de prévention plus classiques soulève cependant de nouveaux défis dans la mesure où il s'agit maintenant de disséminer ces interventions et d'assurer leur pérennité sans retomber dans le piège d'une standardisation qui, en éliminant les processus de co-construction, ferait fi de la nécessité de resituer constamment les programmes dans un espace-temps précis. Diverses communautés se sont approprié les programmes d'expression créatrice, en les transformant à des degrés divers. Ce qui est fascinant dans ces processus d'appropriation, que ce soit par la communauté Attikamek ou par des institutions du Bas St-Laurent, c'est qu'elles mettent en évidence la façon dont les activités créatrices peuvent rejoindre à la fois l'expérience singulière des enfants et les formes d'expression et de transformations collectives qui sont à l'avant-scène dans un environnement précis. On peut se demander dans quelle mesure le développement de programmes de prévention pour les enfants et les jeunes ne devraient pas miser plus souvent sur cette mise en relation des formes de souffrance personnelle et sociale et sur l'émergence de stratégies qui, au-delà de l'individu mais tout en reconnaissant sa place, se fondent sur la consolidation de solidarités.

\section{Conclusion}

En conclusion, un regard sur l'autre, enfant immigrant ou réfugié, vient renouveler notre regard sur nous-mêmes et sur nos enfants. La façon dont la différence de l'autre déstabilise nos savoirs experts met en exergue la violence possible associée aux processus de normalisation et de standardisation qu'ils ont tendance à promouvoir. Comment dès lors peut-on réintroduire la complexité de la personne et du social dans le développement de programmes de prévention? Comment éviter de retomber soit dans une vision clinique individualisante, soit dans une action communautaire ou institutionnelle, certes légitimée par les bonnes intentions de ses acteurs, mais qui évite le questionnement difficile qui surgit inévitablement d'un processus d'évaluation rigoureux? Dans cette perspective les méthodes mises de l'avant par l'approche des données probantes sont utiles à plus d'un titre. En premier lieu, elles permettent parfois de faire une place à des approches alternatives et de leur donner une crédibilité. De plus, elles agissent souvent comme révélateur de nos présupposés au sujet de ce qui est actif dans un programme et des conséquences qui en sont attendues. Enfin, les essais randomisés sont de plus en plus importants pour cerner l'étendue de notre ignorance dans le domaine psychosocial. La reconnaissance du fait que nous en savons très peu est un premier pas essentiel dans les processus de co-construction entourant le développement ou l'adaptation de programmes de prévention pour les enfants et les jeunes.

\section{Références bibliographiques}

Beiser, M., Hou, F., Hyman, I. et Tousignant, M. (2002). Poverty, family process, and the mental health of immigrant children in Canada. American Journal of Public Health, 92(2), 220-227.

Bouchard, G. et Taylor, C. (2008). Fonder l'avenir: le temps de la conciliation. Rapport final de la Commission de consultation sur les pratiques d'accommodement reliées aux différences culturelles. Québec : Gouvernement du Québec.

Bourgeault, G. (1999). Éloge de l'incertitude. Montréal : Bellarmin.

Bourhis, R. , Montreuil, A., Helly, D. et Jantzen, L. (2007). Discrimination et linguicisme au Québec: Enquête sur la diversité ethnique au Canada. Études ethniques au Canada, 39(1-2), 31-49.

Goodman, A., Patel, V. et Leon, D. (2008). Child mental health differences amongst ethnic groups in Britain: a systematic review. BMC Public Health, 8, 258.

Hinton, D. , Chhean, D., Pich, V., Safren, S., Hofmann, S. et Pollack, M. (2005). A randomized controlled trial of cognitive-behavior therapy for Cambodian refugees with treatment-resistant PTSD and panic attacks: A cross-over design. Journal of Traumatic Stress, 18(6), 617-629.

Hörnqvist, M. (2004). The birth of public order policy. Institute of Race Relations, 46(1), 30-52. 
Kleinman, A. et Kleinman, J. (1997). Introduction. Dans A. Kleinman, V. Das et A. Lock (dir.), Social suffering (p. ixxxvii). Berkeley, Los Angeles, London : University of California Press.

Lin, Q. (2001). Toward a caring-centered multicultural education within the social justice context. Education, 122(1), 107-114.

Ngo, V., Langley, A., Kataoka, S. , Nadeem, E., Escudero, P. et Stein, B. (2008). Providing evidence-based practice to ethnically diverse youths: examples from the cognitive behavioral intervention for trauma in schools (CBITS) program. Journal of the American Academy of Child and Adolescent Psychiatry, 47(8), 858-862.

NICE (National Collaborating Centre for Mental Health). (2005). Post-traumatic stress disorder. The management of PTSD in adults and children in primary and secondary care. London : Gaskell / British Psychological Society. (National Clinical Practice Guideline $n^{\circ} 26$ )

Potvin, M., McAndrew, M. et Kanouté, F. (2006). L'Éducation antiraciste en milieu scolaire francophone à Montréal : Diagnostic et prospectives. Rapport de recherche au ministère du Patrimoine Canadien. Montréal : Chaire de recherche du Canada Éducation et rapports ethniques / CEETUM.

Roberts, R., Roberts, C. et Xing, Y. (2006). Prevalence of youth-reported DSM-IV psychiatric disorders among African, European, and Mexican American adolescents. Journal of the American Academy of Child and Adolescent Psychiatry, 45(11), 1329-1337.

Rousseau, C., Benoit, M., Gauthier, M.-F., Lacroix, L., Alain, N., Viger Rojas, M et Bourassa, D. (2007). Classroom drama therapy program for immigrant and refugee adolescents: A pilot study. Clinical child psychology and psychiatry, 12(3), 451-465.

Rousseau, C., Benoit, M., Lacroix, L. et Gauthier, M.-F. (2009). Evaluation of a sandplay program for preschoolers in a multiethnic neighborhood. Journal of child psychology and psychiatry, 50(6), 743-750.

Rousseau, C., Hassan, G., Measham, T. et Lashley, M. (2008). Prevalence and correlates of conduct disorder and problem behavior in West Indian and Filipino immigrant adolescents. European child and adolescent psychiatry, 17(5), 264-273.

Rousseau, C., Hassan, G., Moreau, N. et Thombs, B. (2010). Perceived discrimination and its association with psychological distress in newly arrived immigrants before and after 9/11. American journal of public health, 101(5), 909-915.6.

Rousseau, C. et Jamil, U. (2010). Muslims Families Understanding and Reacting to "The War on Terror". American journal of orthopsychiatry, 80(4), 601-609.

Rousseau, C., Lacroix, L., Singh, A., Gauthier, M.-F. et Benoit, M. (2005). Creative expression workshops in school: Prevention programs for immigrant and refugee children. Canadian child and adolescent psychiatry review, 14(3), 82-85.

Rousseau, C., Singh, A., Lacroix, L., Bagilishya, D. et Measham, T. (2004). Creative expression workshops for immigrant and refugee children. Journal of the American academy of child and adolescent psychiatry, 43(2), 235-238.

Stevens, G. et Vollebergh, W. (2008). Mental health in migrant children. Journal of child psychology and psychiatry, 49(3), 276-294.

Tauvel, J.-P. (1997). L'antiracisme à l'école : Sortir des incantations rituelles. Migrants formation, 109, $184-192$.

Winnicott, D. (1975). Jeu et réalité, l'espace potentiel. Paris : Gallimard. 